EESTI NSV TEADUSTE AKADEEMIA TOIMETISED. 19. KÖIDE
KEEMIA * GEOLOOGIA. 1970, Nr. 4

ИЗВЕСТИЯ АКАДЕМИИ НАУК ЭСТОНСКОИ ССР. ТОМ 19

ХИМИЯ * ГЕОЛОГИЯ. 1970, № 4

\author{
Г. РАЯЛО, ТИИА САВИЧ, В. ДЕМЕНТЬЕВА, АННЕ АННУС, \\ К. ЛЭЭТС
}

\title{
КИНЕТИКА ГИДРОХЛОРИРОВАНИЯ ИЗОПРЕНА
}

Прнсоединение хлористого водорода к кратным связям, т. е. гидрохлорированиє ненасыщенных углеводородов, применяется в разных отраслях промышленности органического синтеза, но несмогря на широкое распространение механизм и кинетика этих реакций пока недостаточно изучены.

В литературе имеются разнообразные и часто даже противоречивые данные о механизме и кинетике присоединения хлористого водорода к кратным связям. Невозможно представнть общую теоретическую схему для всех реакций присоединения. Механизмь реакций зависят от условий проведения процесса и молекулярного строения углеводорода, они также разны для огдельных галогенов и галогенводородов. Кроме того, даже при одннх и тех же реагирующих веществах кинетический механизм и порядок изменяются в зависимости от характера растворителя. Исходя из этого, изучение кинетики реакции нгобходимо в каждом конкретном случае.

Практическое значение изучения присоединения хлористого водорода к изопрену связано с тем, что гидрохлорирование - это одна из стадий нового технологического прощесса получения терпеновых производных на основе продуктов теломеризации изопрена с его гидрохлоридами $\left[{ }^{1}\right]$. Выяснение механизма реакции и определение всех. предэкспоненциальных кинетических констант - основа для проектирования нового технологического процесса, а также для определения оптимальных параметров его проведения.

Рассмотрим только жидкофазную реакцию, так как газофазная реакция между нзопрєном и хлористым водородом в низких температурах и при отсутствии светового излучения, катализаторов и т. д. протекает медленнее по сравнению с жидкофазной.

При гидрохлорировании изопрена образуется смесь из 1,2- и 1,4-аддуктов по следующей сбщей схеме

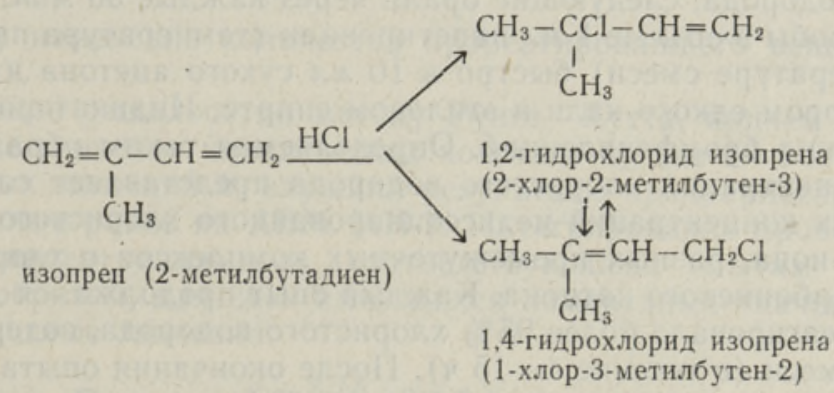

Изучнть кинетику образования 1,2- и 1,4-гидрохлоридов в отдельности трудно, поскольку одновременно с образованием обоих изомеров происходит их взаимная изомеризация $\left[{ }^{2,3}\right]$. Продукт гидрохлорирования изопрена содержит обычно $60-80 \%$ 
1,4-аддукта и 20-40\% 1,2-аддукта. По нашим наблюдениям, содержание 1,2-гидрохлорида в свежеприготовленной смеси выше, чем после длительного хранения. Поэтому кинетика гидрохлорирования нзопрена рассматривается только с точки зрения суммарного содержания гидрохлоридов в смеси.

Проведены опыты по изучению кинетики гидрохлорирования изопрена. Результаты обработаны с помощью электронных вычислительных машин. При этом были проанализированы все возможные из предлагаемых в литературе механизмов реакций присоединения, чтобы выбрать из них самый подходящий для данного случая. Одновременно определялись и самые подходящие численные значения всех кинетических констант.

\section{Методика}

Опыты проводились в стеклянном реакторе периодического действия при стационарном изотермическом режиме. Реактор был снабжен термометром и рубашкой с водой или рассолом для термостатирования. Эксперименты проводились при температурах $30,20,10$ и $4^{\circ} \mathrm{C}$ с разными начальными концентрациями изопрена и его гидрохлоридов в исходной смеси.

Все компоненты для приготовления исходных смесей были очищены ректификацией. Гидрохлориды изопрена выделялись путем ректификации из смеси, полученной в результате гидрохлорирования изопрена сухим хлористым водородом. При составлении исходных смесей варьировались концентрации всех компонентов, но так как в опытах, проведенных при $20^{\circ}$, взаимное соотношение 1,2- и 1,4-гидрохлоридов изопрена заметно не влияло на механизм и скорость присоединения, то в дальнейших опытах на этот фактор не обращалось внимания. Исходная смесь в реакторе сначала охлаждалась до температуры ниже нуля, а затем через нее в течение 4-6 мин пропускался сухой хлористый водород, полученный из концентрированной соляной кислоты под действием концентрированной серной кислоты и пропущенный через осушительную систему. После насыщения хлористым водородом реакционная смесь быстро доводилась до температуры опыта. В дальнейшем реакционный сосуд термостатировали при помощи ультратермостата. В первые минуты после нагревания температура смеси благодаря экзотермичности реакции повышалась, но вскоре выравнивалась за счет хорошего теплообмена. Температура смеси во время опыта поддерживалась с точностью $0,2^{\circ}$.

После достижения нужной температуры, т. е. в начале опыта, из реактора брали первую пробу для определения аналитического содержания хлористого водорода, следующие брали через каждые 30 мин, а затем через час. Пробы в объеме 1 мл пипетировали (температура пипетки равнялась температуре смеси) быстро в 10 мл сухого ацетона и титровали 0,05 н. раствором едкого кали в этиловом спирте. Индикатором при титровании служил бромфенилсиний. Определяемая таким образом аналитическая концентрация хлористого водорода представляет собой сумму молекулярных концентраций недиссоциированного хлористого водорода, водородного иона, разных промежуточных комплексов с хлористым водородом и карбониевого катиона. Каждый опыт продолжался до тех пор, пока не прореагировало более $95 \%$ хлористого водорода, содержащегося в исходной смеси (в течение 4-15 ч). После окончания опыта реакционная смесь подвергалась хроматографическому анализу. По данным опытов были рассчитаны концентрации компонентов в начале и в конце опыта, а также количество прореагировавшего вещества (полученных гидрохлоридов) в зависимости от времени. 


\section{Анализ экспериментальных данных}

Предварительные расчеты по выяснению наиболее вероятных из всех предлагаемых механизмов реакций проводились с помощью аналоговой вычислительной машины (ABM) MH-7. При этом рассматриваемую систему кинетических и балансовых уравнений вместе с начальными условиями моделировали на аналоговой машине, а опытную кривую зависимости количества прореагировавшего вещества от времени аппроксимировали при помощи блока нелинейностей. Затем в случае варьирования при помощи соответствующих потенциометров констант дифференцнальных уравнений в математической модели мы старались найти такие значения констант, при которых кривая, полученная в результате интегрирования, максимально совпадает с кривой аппроксимации экспериментальных данных. Обе кривые вычерчивались на бумаге с помощью двухпозиционного регистрирующего прибора. Сравнивая наилучшие приближения, полученные при разных системах кинетических уравнений, можно выявить, который из проверяемых механизмов реакций наиболее вероятен. Однако класс точности аналоговой машины МН-7 невысок и количество операционных усилителей недостаточно. При моделировании некоторых реакционных схем приходилось вводить серьезные упрощения. Кроме того, сравнение схем реакций и определение констант проводилось по опытам. Поэтому аналоговая вычислительная машина применялась только для предварительных расчетов при выяснении таких механизмов реакций, которые явно не совпадали с результатами опытов.

В окончательных расчетах по определению механизма реакций и кинетических констант применялась ӘЦВМ «Минск-32». Методика расчетов излагается ниже.

Мы располагали экспериментальными данными $j=1 \ldots m$ опытов, каждый из них характеризовался $a_{i}(i=1 \ldots n)$ начальными концентрациями компонентов и проводился при постоянной температуре $(t)$. В каждом опыте определялось количество прореагировавшего вещества $\left(\bar{x}_{i}\right)$ в моменты времени $\left(\tau_{r}\right)$, где $r=0 \ldots R$,

$$
\bar{x}_{t}=f_{1}\left(a_{i j}, \tau_{r}\right) \text {. }
$$

Кроме того, мы имели систему дифференциальных уравнений скоростей, которая соответствует принятому нами механизму реакций и может быть в рассматриваемых случаях приведена к одному общему уравнению

$$
\frac{d x}{d \tau}=f\left(x, k_{s}, a_{i}\right),
$$

выражающему изменение количества прореагировавшего вещества $(x)$ по времени $(\tau)$.

Это уравнение решалось по методу Рунге-Кутта, причем принимались некоторые значения кинетических констант $k_{s}$, где $s=1 \ldots q$. В качестве исходных значений $k_{s}$ служили результаты предварительных расчетов, полученные ранее на АВМ MН-7. Интегрирование проводилось с начальными концентрациями $a_{i j}$, соответствующими опытам при данчной $t$. Таким образом, для всех имеющихся измеренных значений $\bar{x}_{t}$ мы получили расчетные значения

$$
x_{t}=f_{2}\left(k_{s}, a_{i j}, \tau_{r}\right) .
$$

На основе этих данных можно определить значение функции $F(k)$, представляющей собой сумму квадратных отклонений расчетных данных от экспериментальных при данном выборе параметров $k_{s}$, 


$$
F(k)=\sum_{j=1}^{m} \sum_{r=0}^{R}\left(x_{t}-\bar{x}_{t}\right)^{2}
$$

Дальнейшая задача заключалась в том, чтобы варьированием констант $k_{s}$ найти минимум функции $F(k)$. Поиск экстремума проходил па программе, составленной в Институте кибернетики АН ЭССР.

\section{Обсуждение результатов}

Уже в ходе предварительных расчетов выяснилось, что более вероятными оказываются те механизмы реакций, кинетические уравнения которых в итоговом, упрощенном виде показывают квадратную зависимость скорости реакции от концентрации хлористого водорода. С этой точки зрения маловероятным оказался радикальный цепной механизм присоединения, не подходят также чисто ионный механизм реакции и другиепредлагаемые в литературе схемы присоединения, выражающие слишком слабую зависимость скорости реакции от концентрации хлористого водорода. Выяснилось, что в ходе реакции должны образовываться промежуточные комплексы с участием хлористого водорода.

Далее приводятся те механизмы реакций, которые оказались самыми вероятными для рассматриваемого случая. Первый из них учитывает то, что хлористый водород присоединяется к хлоридиону с образованием. комплексного аниона $\mathrm{HCl}_{2}^{-}$, а протон присоединяется к молекуле изопрена с образованием катиона $\mathrm{C}_{5} \mathrm{H}_{9}^{-}\left[{ }^{4}\right]$.

$$
\begin{gathered}
\mathrm{HCl}_{k_{2}}^{\stackrel{k_{1}}{\rightleftarrows}} \mathrm{H}^{+}+\mathrm{Cl}^{-} \\
\mathrm{Cl}^{-}+\mathrm{HCl} \underset{k_{4}}{\stackrel{k_{3}}{\rightleftarrows}} \mathrm{HCl}_{2}^{-} \\
\mathrm{C}_{5} \mathrm{H}_{8}+\mathrm{H}^{+} \underset{k_{6}}{\stackrel{k_{5}}{\rightleftarrows}} \mathrm{C}_{5} \mathrm{H}_{9}^{+} \\
\mathrm{C}_{5} \mathrm{H}_{9}^{+}+\mathrm{HCl}_{2}^{-} \stackrel{k_{7}}{\rightarrow} \mathrm{C}_{5} \mathrm{H}_{9} \mathrm{Cl}+\mathrm{HCl}
\end{gathered}
$$

Систему кинетических и балансовых уравнений, соответствующую этсму механизму реакций, можно привести к виду

$$
\begin{gathered}
\frac{d x}{d \tau}=k_{7}\left[\mathrm{C}_{5} \mathrm{H}_{9}^{+}\right]\left[\mathrm{HCl}_{2}^{-}\right]=\frac{k_{1} k_{3} k_{5} k_{7}}{k_{2} k_{4} k_{6}}\left[\mathrm{C}_{5} \mathrm{H}_{8}\right][\mathrm{HCl}]^{2}, \\
a_{1}-x-[\mathrm{HCl}]-\left[\mathrm{H}^{+}\right]-\left[\mathrm{C}_{5} \mathrm{H}_{9}^{+}\right]=0 \\
a_{2}-x-\left[\mathrm{C}_{5} \mathrm{H}_{8}\right]-\left[\mathrm{C}_{5} \mathrm{H}_{9}^{+}\right]=0
\end{gathered}
$$

Здесь $x$ - количество прореагировавшего вещества;

$a_{1}$ - исходная аналитическая концентрация хлористого водорода;

$a_{2}$ - исходная аналитическая концентрация изопрена;

$\left[\mathrm{C}_{5} \mathrm{H}_{8}\right],[\mathrm{HCl}],\left[\mathrm{C}_{5} \mathrm{H}_{9}^{+}\right],\left[\mathrm{HCl}_{2}^{-}\right],\left[\mathrm{H}^{+}\right]-$концентрации компонентов.

Предполагая, что $\left[\mathrm{C}_{5} \mathrm{H}_{9}^{+}\right] \ll a_{1},\left[\mathrm{C}_{5} \mathrm{H}_{9}^{+}\right] \ll a_{2}$ и $\left[\mathrm{H}^{+}\right] \approx\left[\mathrm{HCl}_{2}^{-}\right]$, полу:чим уравнение скорости в виде 


$$
\begin{gathered}
\frac{d x}{d \tau}=\frac{k_{1} k_{9} k_{5} k_{7}}{k_{2} k_{i} k_{6}\left(1+\sqrt{\frac{k_{1} k_{3}}{k_{2} k_{4}}}\right)^{2}}\left(a_{1}-x\right)^{2}\left(a_{2}-x\right)= \\
=K_{1}\left(a_{1}-x\right)^{2}\left(a_{2}-x\right) .
\end{gathered}
$$

В данных условиях вероятна и вторая схема механизма реакции. В ней учитывается широко распространенная теория образования молекулярного комплекса (л-комплекса) между изопреном и хлористым водородом, который в дальнейшем переходит на б-комплекс или карбониевый катион $[5,6]$. Если и в этом случае принимать во внимание ассоциацию хлорид-иона с молекулой хлористого водорода, получим следующий механизм реакций:

$$
\begin{gathered}
\mathrm{C}_{5} \mathrm{H}_{8}+\mathrm{HCl} \underset{k_{2}}{\stackrel{k_{1}}{\rightleftarrows}} \mathrm{C}_{5} \mathrm{H}_{8} \cdot \mathrm{HCl} \\
\mathrm{C}_{5} \mathrm{H}_{8} \cdot \mathrm{HCl} \underset{k_{4}}{\stackrel{k_{3}}{\rightleftarrows}} \mathrm{C}_{5} \mathrm{H}_{9}^{+}+\mathrm{Cl}^{-} \\
\mathrm{Cl}^{-}+\mathrm{HCl} \underset{k_{6}}{\stackrel{k_{5}}{\rightleftarrows}} \mathrm{HCl}_{2}^{-} \\
\mathrm{C}_{5} \mathrm{H}_{9}^{+}+\mathrm{HCl}_{2}^{-} \underset{k_{7}}{\rightarrow} \mathrm{C}_{5} \mathrm{H}_{9} \mathrm{Cl}+\mathrm{HCl}
\end{gathered}
$$

Кннетические и балансовые уравнения можно привести к виду

$$
\begin{gathered}
\frac{d x}{d \tau}=k_{7}\left[\mathrm{C}_{5} \mathrm{H}_{9}^{+}\right]\left[\mathrm{HCl}_{2}^{-}\right]=\frac{k_{1} k_{3} k_{5} k_{7}}{k_{2} k_{4} k_{6}}\left[\mathrm{HCl}^{2}\left[\mathrm{C}_{5} \mathrm{H}_{8}\right],\right. \\
a_{1}-x-[\mathrm{HCl}]-\left[\mathrm{C}_{5} \mathrm{H}_{8} \cdot \mathrm{HCl}\right]-\left[\mathrm{C}_{5} \mathrm{H}_{9}^{+}\right]=0 \\
a_{2}-x-\left[\mathrm{C}_{5} \mathrm{H}_{8}\right]-\left[\mathrm{C}_{5} \mathrm{H}_{8} \cdot \mathrm{HCl}\right]-\left[\mathrm{C}_{5} \mathrm{H}_{9}^{+}\right]=0
\end{gathered}
$$

Здесь $\left[\mathrm{C}_{5} \mathrm{H}_{8} . \mathrm{HCl}\right]$ - концентрация молекулярного комплекса (л-комплекса).

Считая $\left[\mathrm{C}_{5} \mathrm{H}_{9}^{+}\right] \ll a_{1},\left[\mathrm{C}_{5} \mathrm{H}_{9}^{+}\right] \ll a_{2}$ и $\left[\mathrm{C}_{5} \mathrm{H}_{8} . \mathrm{HCl}\right] \ll a_{2}$, получим

$$
\frac{d x}{d \tau}=\frac{k_{\mathrm{i}} k_{3} k_{5} k_{7}\left(a_{1}-x\right)^{2}\left(a_{2}-x\right)}{k_{2} k_{4} k_{6}\left[\frac{k_{1}}{k_{2}}\left(a_{2}-x\right)+1\right]^{2}}=\frac{K_{1}\left(a_{1}-x\right)^{2}\left(a_{2}-x\right)}{\left[K_{2}\left(a_{2}-x\right)+1\right]^{2}} .
$$

Третий из предлагаемых более вероятных механизмов учитывает то. обстоятельство, что молекула изопрена может образовывать промежуточный комплекс не только с одной, а с двумя и даже тремя молекулами хлористого водорода в зависимости от полярности и других свойств среды, влияющих на сольватацию $[3,7,8]$.

Учитывая это, можно выразить схему реакций в следующем общем виде:

$$
\begin{gathered}
\mathrm{C}_{5} \mathrm{H}_{8}+n \mathrm{HCl} \underset{k_{2}}{\stackrel{k_{1}}{\rightleftarrows}} \mathrm{C}_{5} \mathrm{H}_{8} \cdot n \mathrm{HCl} \\
\mathrm{C}_{5} \mathrm{H}_{8} \cdot n \mathrm{HCl} \underset{k_{4}}{\stackrel{k_{3}}{\rightleftarrows}} \mathrm{C}_{5} \mathrm{H}_{9}^{+}+(n-1) \mathrm{HCl}^{+} \mathrm{Cl}^{-} \\
\mathrm{C}_{5} \mathrm{H}_{9}^{+}+(n-1) \mathrm{HCl} \cdot \mathrm{Cl}^{-} \stackrel{k_{5}}{\rightarrow} \mathrm{C}_{5} \mathrm{H}_{9} \mathrm{Cl}+(n-1) \mathrm{HCl}
\end{gathered}
$$


Считая концентрацию всех промежуточных комплексов ничтожной, лолучим

$$
\begin{aligned}
\frac{d x}{d \tau}= & \frac{k_{1} k_{3} k_{5}}{k_{2} k_{4}}\left(a_{1}-x\right)^{n}\left(a_{2}-x\right)= \\
& =K_{1}\left(a_{1}-x\right)^{n}\left(a_{2}-x\right) .
\end{aligned}
$$

Это уравнение при $n=1$ и $n=3$ маловероятно, а при $n=2$ повторяет конечное уравнение (8) уже рассмотренной нами первой схемы, но существует и возможность одновременного образования комплексов с разным содержанием хлористого водорода. Это приводит к смешанным механизмам реакщий, дающим сводные кинетические уравнения в виде:

$$
\begin{aligned}
& \frac{d x}{d \tau}=K_{1}\left(a_{1}-x\right)^{2}\left(a_{2}-x\right)+K_{2}\left(a_{1}-x\right)\left(a_{2}-x\right), \\
& \frac{d x}{d \tau}=K_{1}\left(a_{1}-x\right)^{2}\left(a_{2}-x\right)+K_{2}\left(a_{1}-x\right)^{3}\left(a_{2}-x\right) .
\end{aligned}
$$

Если в случае $n=2$ учитывать и концентрацию промежуточного комплекса, получим следующее сводное уравнение:

$$
\frac{d x}{d \tau}=K_{1} \frac{1+2 K_{2}\left(a_{1}-x\right)\left(a_{2}-x\right)-\sqrt{1+4 K_{2}\left(a_{1}-x\right)\left(a_{2}-x\right)}}{2 K_{2}^{2}\left(a_{2}-x\right)} .
$$

В таблице приводятся наиболее подходящие значения кинетических коэффициентов $(K)$ и минимальные суммы квадратных отклонений рас'четных данных от экспериментальных $F(k)_{\text {мин. }}$

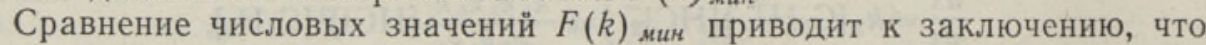
рассмотренные выше три механизма реакций позволяют почти с одинажовой точностью описать кинетику протекания их.

Рассматривая значения приведенных в таблице кинетических констант, можно сделать некоторые выводы.

Если в растворе образуется молекулярный комплекс (л-комплекс) с $\mathrm{HCl}$ по второй рассмотренной нами схеме механизма реакций, то концентрация его должна быть лишь незначительной, так как величина константы $K_{2}$ в формуле (12) невысокая по сравнению с константой $K_{1}$. В случае $K_{2} \approx 0$ уравнение (12) совпадает с уравнением (8).

Если реакция протекает по третьей схеме, то в растворе не образуются комплексы с участием трех молекул хлористого водорода, поскольку константа $K_{2}$ в (15) равна нулю. По этой причине и уравнение (15) совпадает с (8). Что касается уравнения (14), учитывающего образование комплексов с одной молекулой $\mathrm{HCl}$, то надо отметить, что константа $K_{2}$ в этом уравнении имеет определенное значение. Кроме того, это уравнение несколько точнее по сравнению с другими отвечает экспериментальным данным. Это указывает на то, что в случае протекания реакции по третьей схеме изопрен может образовать наряду с комплексами, содержащими две молекулы $\mathrm{HCl}$, комплексы с одной молекулой $\mathrm{HCl}$. Однако относительная доля этих комплексов незначительна и в практических расчетах ее не стоит учитывать. Уравнение (16), учитывающее некоторое содержание (менее 1/10 от содержания $\mathrm{HCl}$ ) промежуточного комплекса в растворе и при $n=2$, не является точнее других формул.

Из сказанного выше следует, что невозможно установить, какой из приведенных выше трех механизмов реакций наиболее вероятен, так как в случае незначительных концентраций промежуточных комплексов и $n=2$ в уравнении (13) они все приводят к одинаковому конечному урав- 


\section{Кинетические константы и суммы квадратных отклонений}

\begin{tabular}{|c|c|c|c|c|c|c|}
\hline (1) & Уравнение & 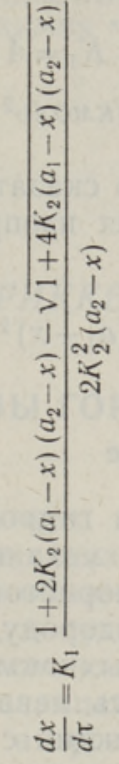 & 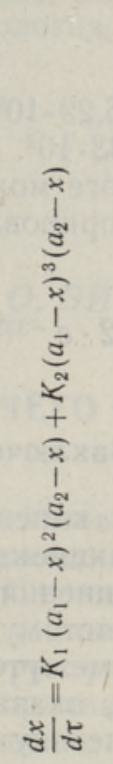 & 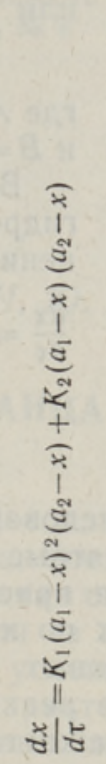 & 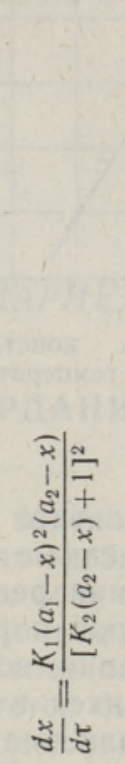 & 竎 \\
\hline 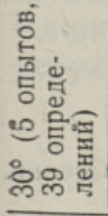 & $\begin{array}{l}K_{1}, \Omega^{2} / м и н \cdot \kappa м о л b^{2} \\
K_{2}, \Omega^{2} / \text { мuн } \cdot \kappa м о л b^{2} \\
F(k)_{\text {мин }} 10^{-9}\end{array}$ & $\begin{aligned} 85550 \\
272500 \\
1,12\end{aligned}$ & $\begin{array}{c}81750 \\
0 \\
1,14\end{array}$ & $\begin{array}{r}72350 \\
0,076 \\
0,91\end{array}$ & $\begin{array}{l}81800 \\
<0,05 \\
1,14\end{array}$ & 81750 \\
\hline 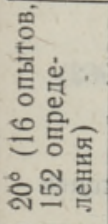 & $\begin{array}{l}K_{1}, \Omega^{2} / \text { мuн } \cdot \kappa м о л b^{2} \\
K_{2}, \Omega^{2} / \text { мuн } \cdot \kappa м о \Omega b^{2} \\
F(k)_{\text {ичн }} 10^{-9}\end{array}$ & $\begin{array}{r}60000 \\
25400 \\
11,53\end{array}$ & $\begin{array}{c}59800 \\
0 \\
11,54\end{array}$ & $\begin{array}{c}52300 \\
0,35 \\
11,0\end{array}$ & $\begin{array}{c}59800 \\
0,000022 \\
11,54\end{array}$ & $\begin{array}{l}59800 \\
11,54\end{array}$ \\
\hline 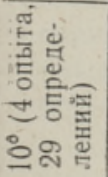 & $\begin{array}{l}K_{1}, \Omega^{2} / \text { мин } \cdot \kappa м о л b^{2} \\
F(k)_{\text {мин }} 10^{-9}\end{array}$ & & & & & $\begin{array}{c}46200 \\
5,47\end{array}$ \\
\hline 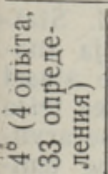 & $\begin{array}{l}K_{1}, \Omega^{2} / \text { мин } \cdot \kappa м о л b^{2} \\
F(k)_{\text {мин }} 10^{-9}\end{array}$ & & & & & $\begin{array}{l}31800 \\
13,64\end{array}$ \\
\hline
\end{tabular}

нению. Формула (8) с достаточной точностью выражает кинетику гидрохлорирования изопрена при постоянной температуре.

Теперь рассмотрим зависимость константы скорости реакции от температуры, предполагая, что все частные коэффициенты в формулах (8), (12) и (13) подчиняются закону Аррениуса $K=A \cdot e^{-E / R T}$ и соотношение $k_{1} k_{3} / k_{2} k_{4}$ в формуле (8) практически равно нулю.

На графике (см. рисунок) приведена зависимость константы $K_{1}$ от температуры в виде $\ln K_{1}=f(1 / T)$. Эта зависимость практически прямолинейна и выражается уравнением: 


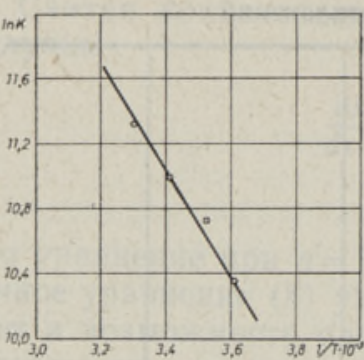

Зависимость константы скорости от температуры.

$$
\ln K_{1}=A^{\prime}-B / T
$$

или

$$
K_{1}=A e^{-B / T},
$$

где $A=5,29 \cdot 10^{9} \Omega^{2} / \kappa м о л b^{2} \cdot м и н=88,2 \mu^{6} / \kappa м о л b^{2} \cdot с е к$ и $B=3,33 \cdot 10^{3}$.

В итоге можно сказать, что скорость реакции гидрохлорирования изопрена выражается уравнением

$$
\frac{d x}{d \tau}=88,2 \cdot e^{-3330 / T}\left(a_{1}-x\right)^{2}\left(a_{2}-x\right) \kappa м о л ь / M^{3} \cdot \text { сек. }
$$

\section{Заключение}

В результате исследования кинетики гидрохлорирования изопрена выяснены самые вероятные реакционные механизмы, согласно которым жндкофазная реакция присоединения хлористого водорода к изопрену имеет второй порядок по хлористому водороду и первый по изопрену. Концентрации возможных промежуточных молекулярных комплексов, образующихся в ходе реакции, оказались невысокими. Приведенное в: статье уравнение выражает кинетику реакции с точностью, достаточной. для практических расчетов.

\section{ЛИТЕРАТ У РА}

1. Л э э с К., Изв. АН ЭССР, Хим. Геол., 17, 355 (1968).

2. Ult é e A. J., J. Chem. Soc., 530 (1948).

3. Gould E. S., Mechanismus und Struktur in der organischen Chemie. Verlag Chemie. Weinheim, 1962.

4. P o cker Y., J. Chem. Soc., 1292 (1960).

5. De w a r M. J. S., Hyperconjugation. Ronald Press. N. Y. 1962.

6. Пе т ро в А. А., Ж. Всесоюз. хим. общества им. Д. И. Менделеева, 7, 332 (1962).

7. He u ble in G., Z. Chem., 6, 201 (1966).

8. He uble in G., Mle jnek E., Z. Chem., 7, 340 (1967).
Ннститут химии
Академии наук Эстонской ССР
Поступила в редакцию 12/II 1970

\section{G. RAJALO, TIIA SAVIC, V. DEMENTJEVA, ANNE ANNUS, K. LĂATS}

\section{ISOPREENI HUDROKLOREERIMISE KINEETIKA}

Esitatakse isopreeni ja vesinikkloriidi vahelise reaktsiooni uurimise tulemusi. Statsionaarsel isotermilisel režiimil sooritatud katsete resultaate analüüsiti diskreetse ja analoograali abil. Selgitati välja kōige tōenäolisemad reaktsioonimehhanismid. Nende alusel jôuti lōppjäreldusele, et vesinikkloriidi liitumine isopreeniga on kloorvesiniku suhtes teise ning isopreeni suhtes esimese järgu reaktsioon. Esitatakse võrrand reaktsioonikiiruse arvutamiseks olenevalt lähteainete kontsentratsioonidest, reageerinud aine hulgast ning temperatuurist, samuti vastavate kineetiliste konstantide arvulised väärtused.

\section{G. RAJALO, TIIA SAVIC, V. DEMENTYEVA, ANNE ANNUS, K. LAATS}

\section{KINETICS OF HYDROCHLORINATION OF ISOPRENE}

The paper deals with the results of an experimental investigation concerning thekinetics of reaction of isoprene with hydrogen chloride. The results of experiments wereanalyzed by means of digital and analog computers. The most probable reaction mechanisms were found out. An equation for estimating the reaction rate in dependence on concentrations of components and on temperature, with the values of kinetic constants, is. presented. 